\title{
Intradiurnal wind variations observed in the lower thermosphere over the South Pole
}

\author{
Y. I. Portnyagin ${ }^{1}$, J. M. Forbes ${ }^{2}$, E. G. Merzlyakov ${ }^{1}$, N. A. Makarov ${ }^{1}$, S. E. Palo ${ }^{2}$ \\ ${ }^{1}$ Institute for Experimental Meteorology, 82, Lenin st., Obninsk, Kaluga region, 249020, Russia \\ ${ }^{2}$ Department of Aerospace Engineering Sciences, Campus Box 429, University of Colorado, Boulder, CO 80303-0429 USA
}

Received: 2 June 1998 / Revised: 15 November 1999 / Accepted: 17 November 1999

\begin{abstract}
The first meteor radar measurements of meridional winds in the lower thermosphere (about $95 \pm 5 \mathrm{~km}$ ), along four azimuth directions: $0^{\circ}, 90^{\circ} \mathrm{E}$, $180^{\circ}$ and $90^{\circ} \mathrm{W}$; approximately $2^{\circ}$ from the geographic South Pole were made during two observational campaigns: January 19, 1995-January 26, 1996, and November 21, 1996-January 27, 1997. Herein we report analyses of the measurement results, obtained during the first campaign, which cover the whole one-year period, with particular emphasis on the transient nature and seasonal behavior of the main parameters of the intradiurnal wind oscillations. To analyze the data, two complementary methods are used: the well-known periodogram (FFT) technique and the $S$-transform technique. The most characteristic periods of the intradiurnal oscillations are found to be rather uniformly spread between about $7 \mathrm{~h}$ and $12 \mathrm{~h}$. All of these oscillations are westward-propagating with zonal wave number $s=1$ and their usual duration is confined to several periods. During the austral winter season the oscillations with periods less than $12 \mathrm{~h}$ are the most intensive, while during summer season the 12-h oscillations dominate. Lamb waves and internal-gravity wave propagation, non-linear interaction of the short-period tides, excitation in situ of the short period waves may be considered as possible processes which are responsible for intradiurnal wind oscillations in the lower thermosphere over South Pole.
\end{abstract}

Key words: Meteorology and atmospheric dynamics (middle atmosphere dynamics; thermospheric dynamics; waves and tides)

\section{Introduction}

Few papers exist which describe the specific features of the winds in the mesosphere/lower thermosphere (MLT) region in the vicinity of the rotational poles of the Earth. Among these are the pioneering works of Hernandez et al. (1992a, b, 1993, 1995). Several different types of day-to-day and intra-diurnal (6-24 h) wind oscillations are discussed in these papers. The observational results in these works are based upon optical observations of winds and $\mathrm{OH}$-emission rates near the mesopause and thus were limited in their ability to perform long-term continuous measurements, especially during periods of significant sun and moon illumination of the sampling volume.

The installation of a meteor radar at the South Pole and its successful operation without interruption during two observational campaigns; January 19, 1995-January 26, 1996, and November 21, 1996-January 27, 1997, provided unique opportunities for investigation of a broad spectrum of waves in the wind field in the lower thermosphere over the South Pole for different seasons (Forbes et al., 1995; Portnyagin et al., 1996; Portnyagin et al., 1997, 1998; Palo et al., 1998). In these papers the main features in the seasonal behavior of the different wind oscillations at an average height of $95 \mathrm{~km}$ over the South Pole with characteristic periods equal to or greater than $12 \mathrm{~h}$ are discussed in detail. However, oscillations with periods less than $12 \mathrm{~h}$ were beyond the scope of these papers. Meanwhile, the subdiurnal oscillations in the MLT region near the South Pole appears to be a common feature of this region. Analyzing the results of MLT wind measurements made from August 1, 1991 to August 25, 1991, from August 1, 1992 to August 13, 1992 and from June 19, 1995 to June 30, 1995, Hernandez et al. (1992a, 1995, 1996) show the presence of an oscillation with a period close to $10 \mathrm{~h}$, propagating westward and have ascribed this to a zonal wave number one Lamb wave. In addition, during these campaigns, as well as in the campaign from August 13 to August 23, 1996 (Hernandez et al., 1997) additional 
oscillations with periods near $12 \mathrm{~h}$ were detected. These quasi- $12 \mathrm{~h}$ oscillations were tentatively identified as zonal wave number one inertio-gravity modes of atmospheric oscillation (see Longuet-Higgins, 1968).

We present a view of the intradiurnal wind oscillations in the MLT region over the South Pole, which results from analyses of our meteor radar measurements, with particular emphasis on the seasonal behavior of the main parameters of these oscillations. Meanwhile, the summer-time 12-h wind oscillations are beyond our scope remit, as these oscillations are discussed in detail in another paper (Portnyagin et al., 1998).

\section{The meteor radar system}

The meteor radar system which was used to make the neutral wind measurements we present, is described by Portnyagin et al. (1997a, b) and Palo et al. (1998). The radar operates in a monostatic configuration with four antenna, directed in the horizontal plane along the $0^{\circ}, 90^{\circ} \mathrm{E}, 180^{\circ}$ and $90^{\circ} \mathrm{W}$ geographical meridians. The hourly mean winds, which are assumed to be horizontal, represent Gaussian-weighted averages centered near $95 \mathrm{~km}$ above sea level.

\section{Data analysis procedures}

The gaps cover about $7 \%$ of full data set and are distributed more or less randomly. We have used a leastsquares fitting procedure by a second order polynomial to fit the gaps.

To analyze the data, two complementary methods are used: the well-known periodgram (FFT) and the $S$-transform technique (Stockwell et al., 1996; Fritts et al., submitted 1997). The periodgram reveals the energy presented at discrete frequencies in the data set, supposing that these oscillations are stationary during the period to be analyzed. The transient nature of the oscillations become evident when $S$-transform technique is used. The $S$-transform is closely related to the continuous wavelet transform (CWT), but its localizing function (Gaussian) does not have zero mean (Stockwell et al., 1996; Fritts et al., 1998). The advantage of this method is its direct connection with a spectral Fouriertransformation and the better temporal and frequency resolution, in comparison with the short time Fourier transform. In addition, the $S$-transform permits determination of the absolutely referenced phase $(\mathrm{F})$ of the considered oscillation assuming it is a nonstationary process, while the CWT gives only locally referenced phase information (Stockwell et al., 1996). Knowing the phases of the considered oscillations for different measurement directions gives information on the longitudinal structure of these oscillations. More detail on the $S$-transform can be found in the Appendix.

The hourly wind data for each direction were first filtered using a high-frequency filter with the cut-off period at $36 \mathrm{~h}$ and a low-frequency filter with a cut-off period at $4 \mathrm{~h}$. Then for each sequential interval with duration equal to $512 \mathrm{~h}$ the periodgram and $S$-transform were calculated (with the natural exception at the beginning and the end of the whole data set). Special attention was devoted to the period from June 19 to June 30, 1995 (see later), when the length of analyzed data set was $256 \mathrm{~h}$. The oscillation parameters obtained were analyzed for each measurement direction and then were averaged for all directions. The method of estimation of the significance level for the estimated parameters of the wind oscillations is presented in the Appendix. A modified procedure was carried out for intervals when the regular 12-h oscillation was dominating the wind field (Forbes et al., 1995; Portnyagin et al., 1997). We increased a constant $c$ in $S$-transform procedure (see Appendix), which is responsible for frequency resolution. It provides the possibility to determine the frequencies of other oscillations with smaller amplitudes.

\section{Results}

From June 19, 1995, to June 30, 1995, simultaneous observations were made using the meteor radar and collocated $\mathrm{OH}$ spectrometer at the South Pole. This has provided an opportunity to compare the wind measurements made from the two instruments. Some results from these simultaneous optical and radar wind measurements have already been published (Hernandez et al., 1996). As noted, despite the difference in the measuring heights (about $87 \mathrm{~km}$ for the optical device and $95 \mathrm{~km}$ for meteor radar) the correlation coefficient between two data sets is $r=0.82$, which was found to be statistically significant to the $99 \%$ confidence level.

We begin our presentation with a more detailed analysis of the meteor radar wind data for this particular period. The periodgrams for all measurement directions and for the data, averaged over four directions, are shown in Fig. 1. In this figure the 95\% significance levels are drawn separately for three successive largest peaks as Whittle's test (Whittle, 1951). We used 64 spectral peaks, which were retained after filtering. For the averaged spectrum it is more reasonable to show the single significance level covering all of the most prominent peaks. This level corresponds to $95 \%$ significance level of the largest peak. The probability, that two or more spurious peaks would be above the level is significantly less than 0.05 (Whittle, 1951). It can be seen that the relative power of a given frequency oscillation is not the same for the different directions. Moreover, some oscillations may be considered as significant for a certain direction and as a not significant for another. The same difference between different directions is seen in optical data, hence it is presumed to have a geophysical origin.

There are several reasons for this. They include differences in the sampling statistics for different directions; frequency spectrum and background noise levels for different directions. In addition there are some background oscillations, perhaps as observed by Hernandez et al. (1997) and Palo et al. (1998), which may modulate the waves considered mainly in one direction and distort the spectrum. 

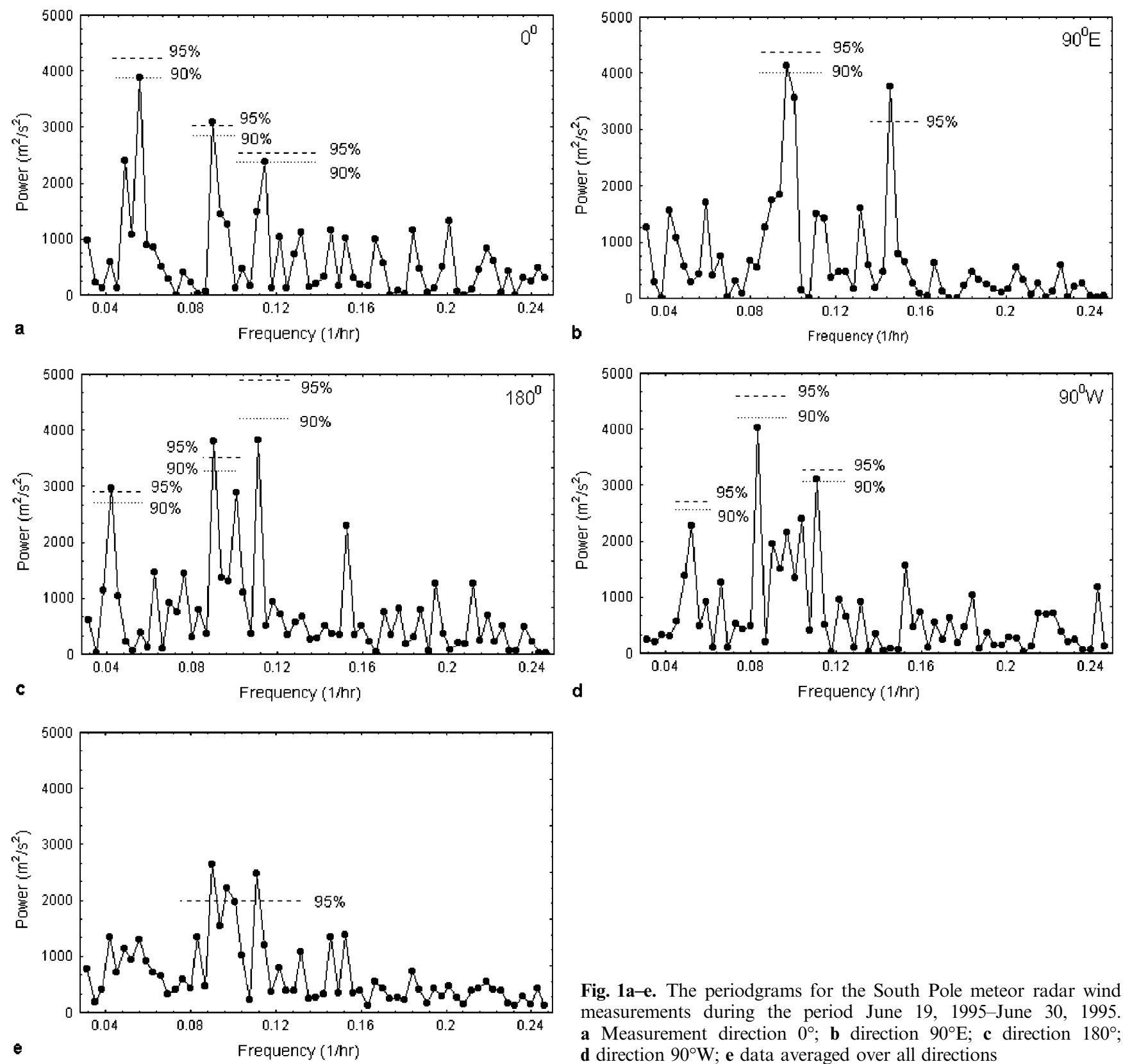

Fig. 1a-e. The periodgrams for the South Pole meteor radar wind measurements during the period June 19, 1995-June 30, 1995. a Measurement direction $0^{\circ}$; b direction $90^{\circ} \mathrm{E}$; c direction $180^{\circ}$; d direction $90^{\circ} \mathrm{W}$; e data averaged over all directions

To be sure of the significance of the results we have used the estimation of the power spectrum as an average one for all directions. The averaged periodgram shows some definite peaks, which are consistent with those derived from optical data analysis (Hernandez et al., 1996). The oscillations with periods equal to $11.1 \mathrm{~h} \pm$ $0.4(10.9 \mathrm{~h}), \quad 10.3 \mathrm{~h} \pm 0.4(10.36 \mathrm{~h})$ and $9 \mathrm{~h} \pm 0.3$ $(9.9 \mathrm{~h})$ deserve attention. In parentheses we point out the corresponding periods, arrived at in the analysis by Hernandez et al. (1996). An additional argument in favor of geophysical significance of the selected peaks is the phase behavior of the corresponding wind oscillations (see later). There are several reasons why our results give slightly different results from those of Hernandez et al. (1996). They include: the Hernandez results correspond to $87 \mathrm{~km}$, ours to $95 \mathrm{~km}$, our data includes four azimuth directions, while the Hernandez

results were received from periodogram for one direction (actually his measurements were carried out along eight azimuths); the volume sample statistics are different for optical and radar methods; the background noise level is different for different methods and there is different frequency resolution for different methods. Results of the $S$-transform analysis for this campaign are shown in Fig. 2 in a dimensionless form (the wind velocities were first divided by $19 \mathrm{~m} / \mathrm{s}$ ). It can be easily seen that the selected oscillations reveal a very definite transient nature and are mainly separated by their time of appearance. Such transient behavior for the wind oscillations over the South Pole with periods of $11.6 \mathrm{~h}$ and $10 \mathrm{~h}$ has been noticed earlier (Hernandez et al., 1995; Hernandez et al., 1997). Usually each oscillation is significant only for several periods of its existence. The time resolution of the $S$-transform results is about one 

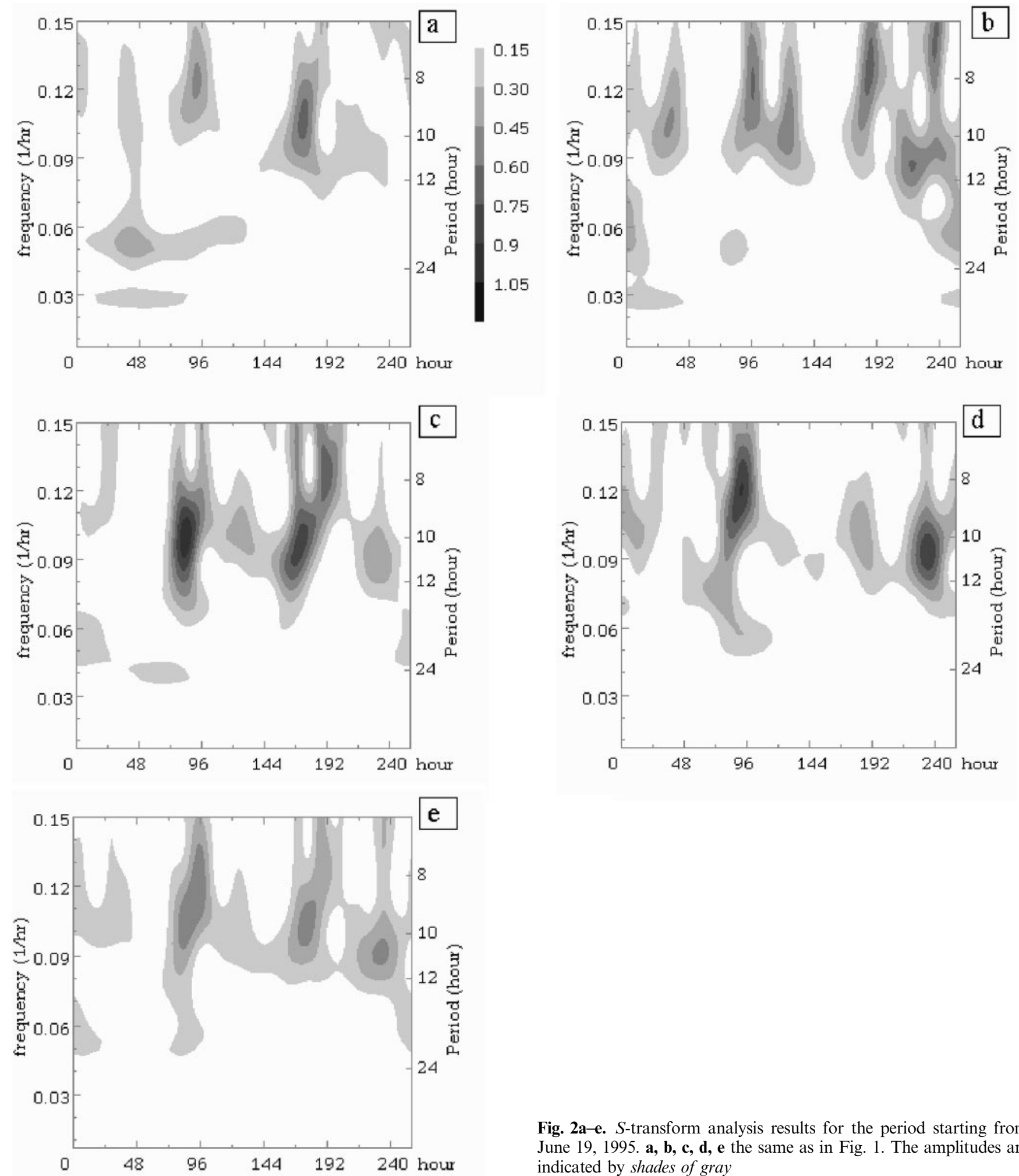

period of an oscillation, then the amplitude of oscillation may attain its maximum value at different moments for different directions. As in Fig. 1, the relative strength of these oscillations is rather different for different directions. From the $S$-transform results the significant periods are found to be: $10.7 \pm 1.5 \mathrm{~h}, 9.5 \pm 1.5 \mathrm{~h}$ and $8.4 \pm 1.4 \mathrm{~h}$, where a resolution is defined as minimum separation between two peaks, which are equal in

Fig. 2a-e. $S$-transform analysis results for the period starting from June 19, 1995. a, b, c, d, e the same as in Fig. 1. The amplitudes are indicated by shades of gray

strength. There is some difference between these values and those obtained using the periodogram technique, however taking into consideration the frequency resolution of $S$-transform we have concluded that this difference is not significant. The peak amplitudes of these oscillations are: $14.6 \mathrm{~m} / \mathrm{s}(6 \mathrm{~m} / \mathrm{s}), 16.1 \mathrm{~m} / \mathrm{s}(5.8 \mathrm{~m} / \mathrm{s})$ and $14.6 \mathrm{~m} / \mathrm{s}(5.7 \mathrm{~m} / \mathrm{s})$, respectively (in parenthesis we note the corresponding amplitude estimated from the 


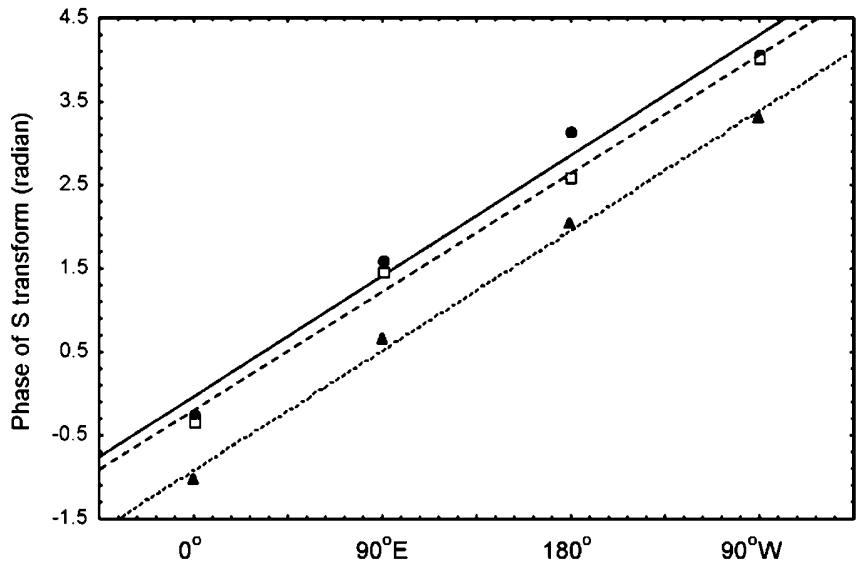

Fig. 3. The relative phases of significant intradiurnal oscillations versus longitude for the observational period June 19, 1995-June 30, 1995. Solid line $8.3 \mathrm{~h}$ oscillation, dashed line $9.5 \mathrm{~h}$ oscillation, dotted line $10.7 \mathrm{~h}$ oscillation

periodgram). Their relative initial phases (not time of maximum) for the different directions are depicted in Fig. 3. For each wave the phases were measured at the moment a wave has its maximum amplitude. From Fig. 3 the conclusion can be made that all these oscillations are westward propagating with the zonal wave number $s=1$. The good fitting of the phases for each oscillation to the straight line, which corresponds to an $s=1$ oscillation, gives additional support to the geophysical significance of the oscillations under discussion.

Other examples of the $S$-transform analysis are shown in Fig. 4. It can easily be seen that for different months (only amplitudes greater than $10 \mathrm{~m} / \mathrm{s}$ are shown) the intradiurnal oscillations are very sporadic in nature. The usual duration of these oscillations is confined to several periods of oscillation. More detailed information about the observed wave events for the observational period since the end of February, 1995 to the end of September, 1995 (period I) is summarized in Table 1. From this Table 1, we may conclude that in spite of the rather broad spread of the periods, the tendency is toward a general decrease in the observed periods during winter months. It is important to note that all significant intradiurnal oscillations, shown in Table 1, are westward propagating with zonal wave number $s=1$. One can see in Fig. 5, that the some tendencies exist to concentrate the wave periods into intervals $10-11 \mathrm{~h}$ and 8-9 h. This histogram is based on the Table 1.

The period from the beginning of October to the beginning of February (period II) is characterized by a strong semidiurnal oscillation (Forbes et al., 1995; Portnyagin et al., 1997). This oscillation masks the other weaker oscillations. As a result, the determination of the parameters of intradiurnal variations during period II are not as accurate as for period I. Figure $4 \mathrm{e}$, d illustrates this situation. In Fig. 4e the results of $S$-transform analysis of the hourly mean data for the February, 1995 are shown. It can be seen from this figure that the very strong semidiurnal oscillation ceases after February 10 (hour 288). Following this time the oscillation with a period of about $9 \mathrm{~h}$ appears with a
Table 1.

\begin{tabular}{|c|c|c|c|c|}
\hline Number & $\begin{array}{l}\text { Period } \\
\text { (hours) }\end{array}$ & $\begin{array}{l}\text { Amplitude } \\
(\mathrm{m} / \mathrm{s})\end{array}$ & $\begin{array}{l}\text { Time of } \\
\text { maximum }\end{array}$ & $\begin{array}{l}\text { Halfwidth } \\
\text { (hours) }\end{array}$ \\
\hline 1 & 12 & 27.6 & Till 02.11 .95 & \\
\hline 2 & 10.4 & 23 & 02.22 & 23 \\
\hline 3 & 10.4 & 13.4 & 02.26 & 18 \\
\hline 4 & 12 & 15 & 02.26 & 31 \\
\hline 5 & 11.4 & 14 & 03.3 .95 & 42 \\
\hline 6 & 10 & 13.5 & 03.7 & 20 \\
\hline 7 & 11.4 & 15.2 & 03.9 & 31 \\
\hline 8 & 12 & 21.2 & 03.12 & 43 \\
\hline 9 & 11.4 & 21.6 & 03.12 & 25 \\
\hline 10 & 11.1 & 12.7 & 03.21 & 23 \\
\hline 11 & 12 & 16.1 & 03.23 & 23 \\
\hline 12 & 10.9 & 14 & 03.30 & 23 \\
\hline 13 & 7.3 & 13.3 & 04.6 .95 & 17 \\
\hline 14 & 6.8 & 12.4 & 04.10 & 17 \\
\hline 15 & 8.3 & 13 & 04.12 & 17 \\
\hline 16 & 9.5 & 15.1 & 04.28 & 17 \\
\hline 17 & 9.1 & 19.4 & 05.6 .95 & 14 \\
\hline 18 & 6.8 & 13.3 & 05.11 & 14 \\
\hline 19 & $7.2-7.5$ & 12.4 & 05.19 & 31 \\
\hline 20 & 10.2 & 14.5 & 06.1 .95 & 23 \\
\hline 21 & 10 & 16.7 & 06.5 & 31 \\
\hline 22 & 8.8 & 24.5 & 06.10 & 17 \\
\hline 23 & 8.3 & 16.1 & 06.22 & 20 \\
\hline 24 & 9.5 & 14.6 & 06.26 & 28 \\
\hline 25 & 10.7 & 14.6 & 06.29 & 28 \\
\hline 26 & 6.9 & 15.4 & 07.8 .95 & 20 \\
\hline 27 & 7.3 & 19.3 & 07.12 & 14 \\
\hline 28 & 8.5 & 17.8 & 07.14 & 23 \\
\hline 29 & 8.4 & 13.1 & 07.22 & 20 \\
\hline 30 & 9.5 & 15 & 07.26 & 25 \\
\hline 31 & 7.9 & 19.1 & 07.30 & 14 \\
\hline 32 & 8.3 & 26.4 & 08.2 .95 & 11 \\
\hline 33 & 9.3 & 22 & 08.22 & 20 \\
\hline 34 & 8.7 & 16.4 & 08.26 & 14 \\
\hline 35 & 11.1 & 12.9 & 09.3 .95 & 34 \\
\hline 36 & 11.1 & 21.1 & 09.11 & 17 \\
\hline 37 & 10.7 & 14.6 & 09.27 & 20 \\
\hline 38 & 10.9 & 11.9 & 10.8 .95 & 31 \\
\hline 39 & 12 & 15 & 10.12 & 37 \\
\hline
\end{tabular}

rather strong maximum on February 22 and a weaker maximum on February 26. Special methods must be developed for correct determination of the parameters of intradiurnal oscillations other than the strong semidiurnal oscillation, observed during period II. One possibility is to use a constant greater than 2 in the $S$-transform procedure. Then we will lose in temporal resolution, but increase the frequency resolution. Independent of the type of analysis applied during summer period, we have detected significant oscillations with periods definitely exceeding $12 \mathrm{~h}(13-14 \mathrm{~h})$.

\section{Discussion and conclusions}

Hernandez et al. (1992a, b) have shown that there is no resemblance between the main parameters of the oscillations at the mesopause heights near the South Pole in the wind field and in the pressure, temperature and density fields. The most important difference is that the typical wind oscillations are characterized by a zonal wave number $s=1$, while the oscillations of the 

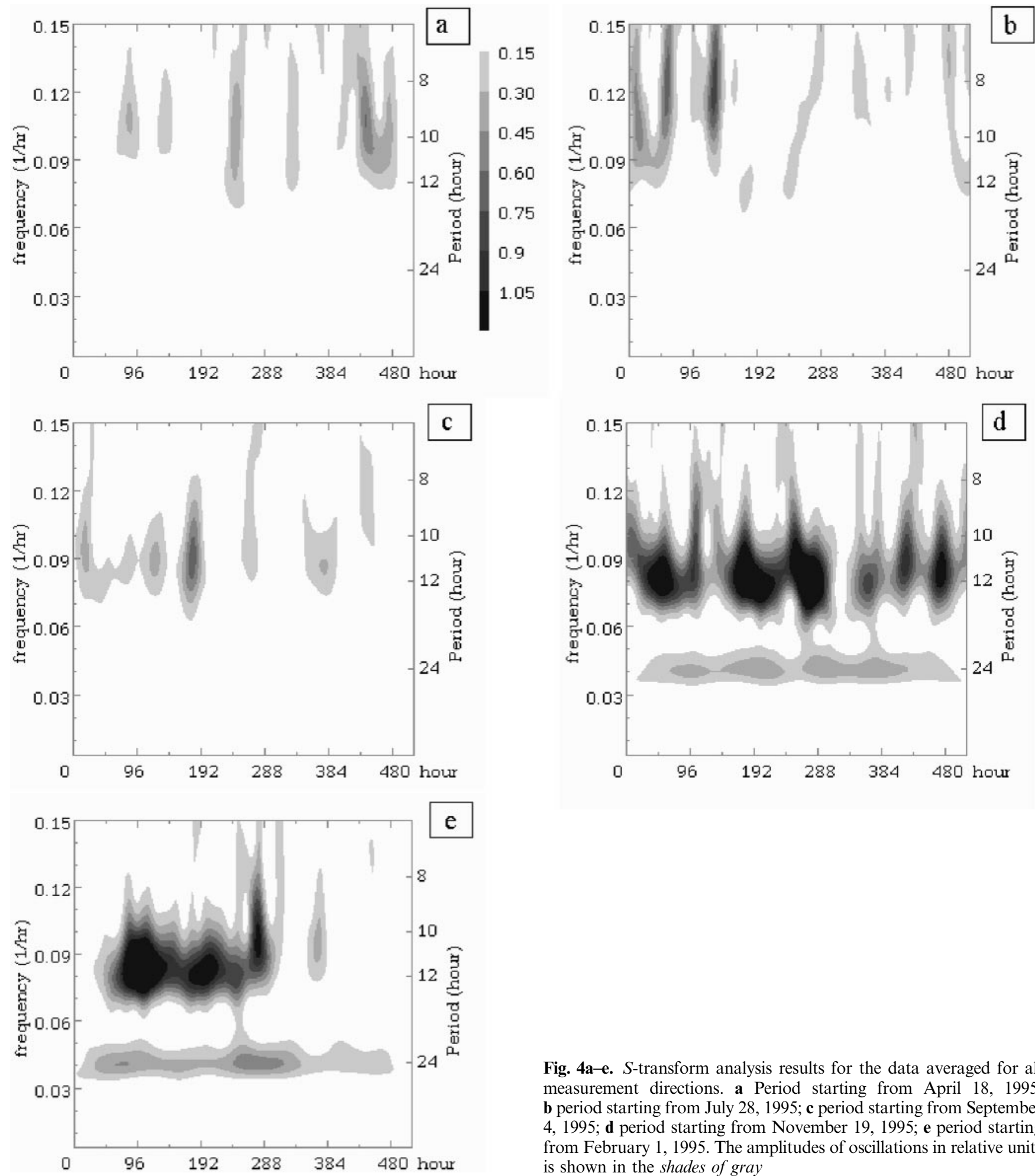

thermodynamic parameters are characterized by a zonal wave number $s=0$. Simultaneous wind and temperature measurements do not show any common peaks in their variations (Hernandez et al., 1992a, b). Hence, the consideration of the intradiurnal temperature and density variations, detected in the upper atmosphere over the South Pole (Collins et al., 1992; Sivjee and Waltersheid, 1994) is beyond the scope of this work.

Fig. 4a-e. $S$-transform analysis results for the data averaged for all measurement directions. a Period starting from April 18, 1995; b period starting from July 28, 1995; c period starting from September 4, 1995; d period starting from November 19, 1995; e period starting from February 1, 1995. The amplitudes of oscillations in relative units is shown in the shades of gray

In discussing the nature of oscillations close to $10 \mathrm{~h}$, detected simultaneously in the upper mesosphere at the South Pole and Scott Base station $\left(78^{\circ} \mathrm{S}\right)$ during several observational campaigns, Hernandez et al. (1992b, 1995, 1996) have concluded that the most plausible explanation for this oscillation is that it is a zonal wave number one Lamb wave, with a meridional mode of number two. This conclusion was based on estimation of the vertical wavelength of this oscillation, found to be 


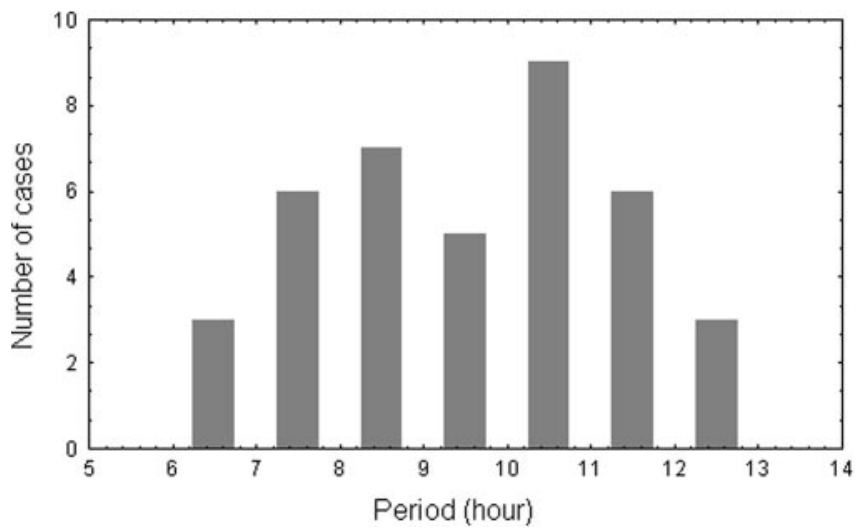

Fig. 5. A histogram of intradiurnal wave periods observed from January 19, 1995 to January 26, 1996

about $100 \mathrm{~km}$, from the Scott Base observations and on the identification of this oscillation from the South Pole measurements. It is worthwhile noting that according to Lindzen and Blake (1972), Lamb waves below about $80 \mathrm{~km}$ are vertically evanescent, but above $80-90 \mathrm{~km}$ their behavior more closely resembles a vertically propagating internal gravity wave (IGW). The existing numerical calculations of the vertical phase structure in the transition region between 80 and $100 \mathrm{~km}$ are highly idealized (Lindzen and Blake, 1972). Additionally experimental determinations of the vertical wavelength of about $100 \mathrm{~km}$ were estimated, using the data from Scott Base within a relatively narrow height interval $(80-100 \mathrm{~km})$. So, the determined wavelength for this oscillation may not be considered as a crucial argument in favor of its identification as a Lamb wave.

From Table 1 it may be inferred that the intradiurnal oscillations with periods exceeding $12 \mathrm{~h}$ are not typical of the upper mesosphere/lower thermosphere over the South Pole. The most characteristic periods of the oscillations are found to be rather uniformly spread between about $7 \mathrm{~h}$ and $12 \mathrm{~h}$. It is interesting to note that oscillations with periods close to those of different tidal modes are not dominant in our results. Also having in mind the fact that all of the observed oscillations have zonal wave number one, we may conclude that the pseudo-tide mechanism, proposed by Walterscheid et al. (Walterscheid et al., 1986; Sivjee et al., 1994), even if possible, is not the main mechanism which gave rise to the observed oscillations.

There is a class of solutions of the Laplace's tidal equation which may be attributable to the considered oscillations. According to Longuet-Higgins (1968) these are the westward traveling modes of type I with $s=1$ for the positive values of dimensionless parameter $\varepsilon=4 \Omega^{2} R^{2} / g h$, involving the rate of rotation $\Omega$, the radius of the globe $R$, the acceleration of gravity $g$ and the equivalent depth $h$. Lindzen and Blake (1972) have shown that for realistic distributions of temperature and dissipation Lamb-like waves do exist, that should therefore dominate the atmospheric response to various lower atmosphere excitations. The equivalent depth for these waves is $9.95 \mathrm{~km}$ i.e., $\varepsilon$ is about 9 . Then we may determine from Longuet-Higgins's (1968) tables the following periods of Lamb-like oscillations with $s=1$ : $10.8 \mathrm{~h}, 8.96 \mathrm{~h}$ and $7.5 \mathrm{~h}$, which correspond to meridional indices $n-s=1,2,3$. These periods are close to those observed. Actually there are meridional inhomogenities in distributions of the parameters of the real atmosphere and a background wind, which are absent in the idealized model of Longuet-Higgins (1968). Additionally the atmospheric parameters are seasonally changing. This is why we cannot expect exact correspondence.

Inertia-gravity wave propagation, non-linear interaction of the short-period tides, excitation in situ of the short period waves may also be considered as possible processes which are responsible for intradiurnal wind oscillations in the lower thermosphere over the South Pole. However due to the limited number of measurement parameters, we do not have solid reasons to discuss the possible roles of these processes in the dynamics of the near-pole region.

Acknowledgements. This work was partly supported by National Science Foundation (Grant OPP-9320879) and by Russian Fund for Fundamental Researches (Grant N 98-05-64014).

Topical Editor F. Vial thanks M.J. Jarvis and D. Riggin for their help in evaluating this paper.

\section{Appendix}

Here we consider some features of the $\boldsymbol{S}$-transform, using a simple form of the input signal. Let the signal $h(t)$ have the form

$h(t)=\exp \left(-t^{2} / 2 \sigma^{2}\right) \cos \left(2 \pi f_{0} t\right)$,

where $f_{0}$ is signal frequency, $\sigma$-a parameter determining the width (duration) of the signal. The $S$-transform is defined as following

$$
\begin{aligned}
S(\tau, f)= & \sqrt{\frac{2}{c}} \int_{-\infty}^{\infty} h(t) \frac{|f|}{\sqrt{2 \pi}} \exp \left\{-\frac{(\tau-t)^{2} f^{2}}{c}\right\} \\
& \times \exp \{-i 2 \pi f t\} \mathrm{d} t
\end{aligned}
$$

A constant $c$ is responsible for the time and frequency resolution. We use $c=2$ for our data set excluding the summer months, when we make $c=4$ for better frequency resolution. For $c=2$ the half amplitude width of the Gaussian localizing function is about 2.4 cycles of the frequency being fitted.

The real part of the response as function of time $\tau$ and response frequency $f$ is equal to:

$$
\begin{aligned}
I_{1}(\tau, f)= & \frac{|f|}{\sqrt{2 \pi}} \frac{1}{2} \exp \left(-\frac{\tau^{2}}{2 \sigma^{2}} \frac{f^{2} \sigma^{2}}{1+f^{2} \sigma^{2}}\right) \\
& \times \cos \left[2 \pi\left(f-f_{0}\right) \tau \frac{f^{2} \sigma^{2}}{1+f^{2} \sigma^{2}}\right] \sqrt{2 \pi \frac{f^{2} \sigma^{2}}{1+f^{2} \sigma^{2}}} \\
& \times \exp \left(-2 \pi^{2} \frac{\left(f-f_{0}\right)^{2} \sigma^{2}}{1+f^{2} \sigma^{2}}\right)+\frac{|f|}{\sqrt{2 \pi}} \frac{1}{2} \\
& \times \exp \left(-\frac{\tau^{2}}{2 \sigma^{2}} \frac{f^{2} \sigma^{2}}{1+f^{2} \sigma^{2}}\right) \\
& \times \cos \left[2 \pi\left(f+f_{0}\right) \tau \frac{f^{2} \sigma^{2}}{1+f^{2} \sigma^{2}}\right] \\
& \times \sqrt{2 \pi \frac{f^{2} \sigma^{2}}{1+f^{2} \sigma^{2}}} \exp \left(-2 \pi^{2} \frac{\left(f+f_{0}\right)^{2} \sigma^{2}}{1+f^{2} \sigma^{2}}\right)
\end{aligned}
$$

The imaginary part $\left(I_{2}\right)$ of the response may be obtained by replacing cos with sin in the above expression. Within the accuracy 
of order $\exp \left(-8 \pi^{2} f_{0}^{2} \sigma^{2} /\left(1+f_{0}^{2} \sigma^{2}\right)\right)$ the maximum of the amplitude is attained at the point $f=f_{0}$. With the same order of accuracy the amplitude of $S$-transform at the point $f=f_{0}$ is equal to

$I_{1}^{2}+I_{2}^{2}=0.25 \exp \left(-\frac{t}{\sigma^{2}} \frac{f_{0}^{2} \sigma^{2}}{1+f_{0}^{2} \sigma^{2}}\right) \frac{f_{0}^{2} \sigma^{2}}{1+f_{0}^{2} \sigma^{2}}$

We consider only positive value of $f$ and $f_{0}$ (assuming $f_{0} \neq 0$ ), therefore we have obtained only half amplitude of the signal, as can be seen from Eq. (3). This is the reason for the appearance in Eq. (4) of a factor 0.25 . Hence, distortion of the duration and the maximum amplitude of the signal depends on the parameter $f_{0} \sigma$, which determines the ratio of the length of the periodic part of the signal to its duration. Because $\sigma$ is a finite value, the duration is overestimated, while the signal amplitude is underestimated. There is no error if a signal has a sinusoidal form. For considered signal form the ratio of the actual signal maximum amplitude to the transformed one is equal to $\sqrt{f_{0}^{2} \sigma^{2} / f_{0}^{2} \sigma^{2}-1}$. This factor is less then $10 \%$ for our data set.

The significance criteria of spectral maxima may be constructed in accordance with Walker's significance test in harmonic analysis (Davis, 1941). Let us suppose that the input signal in any moment of time $t_{k}$ is a stochastic value with a normal (Gaussian) distribution $(0, \sigma)$. The discrete $S$-transform may be divided into the two sums, real $S_{1}$ and imaginary $S_{2}$ :

$S_{j, n}=\frac{1}{N} \sum_{k=0}^{N-1} h(k) \frac{j}{\sqrt{2 \pi}} \exp \left[-\frac{(n-k)^{2} j^{2}}{2 N^{2}}\right]\left[\cos \frac{2 \pi j k}{N}-i \sin \frac{2 \pi j k}{N}\right]$,

where $i$ is an imaginary unit. $S_{1}$ and $S_{2}$ are also normally distributed with the parameters $E\left(S_{1}\right)=E\left(S_{2}\right)=0$,

$\operatorname{Var}\left(S_{1}\right)=\frac{j^{2}}{2 \pi N^{2}} \sigma^{2} \sum_{k=0}^{N-1} \exp \left[-\frac{(n-k)^{2} j^{2}}{N^{2}}\right] \cos ^{2} \frac{2 \pi j k}{N}$

$\operatorname{Var}\left(S_{2}\right)=\frac{j^{2}}{2 \pi N^{2}} \sigma^{2} \sum_{k=0}^{N-1} \exp \left[-\frac{(n-k)^{2} j^{2}}{N^{2}}\right] \sin ^{2} \frac{2 \pi j k}{N}$

Near the frequencies of interest to us of order 0.1 it appears that for the time steps $\mathrm{n}$ in the limits $20<n<490$ $\operatorname{Var}\left(S_{1}\right)=\operatorname{Var}\left(S_{2}\right)=j-/(4 N \sqrt{\pi}) \sigma^{2}$ with an error less than $1 \%$ and $\operatorname{Cov}\left(S_{1}, S_{2}\right)=0$. Let us note that the $S$-transform distorts the spectral content of the data namely at the beginning and the end of the data sequence. Thus, the value $\left(S_{1}^{2}+S_{2}^{2}\right) / \operatorname{Var}\left(S_{1}\right)$ for a given discrete frequency, determined by $j$, is characterized by $\chi^{2}$-distribution with two degrees of freedom. Respectively, for a case of averaging over four measurement directions we have $\chi^{2}$, a distribution with 8 degrees of freedom, if it is assumed that the variances for the different directions are equal. We assume that the variance for the time sequence $h\left(t_{k}\right)$ is known before applying the $S$-transform. To obtain the variance we use the corresponding nonfiltered data set without trend. The number of independent peaks is estimated as a ratio of the full length of the observation period to double period of the oscillation. Thus using this algorithm we can estimate the $95 \%$ significance level for any frequency.

\section{References}

Collins, R. L., D. C. Senft, and C. S. Gardner, Observations of a 12-h wave in the mesopause region at the South Pole, Geophys. Res. Lett., 19, 57, 1992.

Davis, H. T., The analysis of economic time series, The Cowles Commission for Research in economics. Monograph 6, Bloomington, Indiana, 1941.

Forbes, J. M., N. A. Makarov, and Y. I. Portnyagin, First results from the meteor radar at South Pole: a large 12-hour oscillation with zonal wavenumber one, Geophys. Res. Lett., 22(23), 32473250, 1995.

Fritts, D. C., D. M. Riggin, B. B. Balsley, and R. G. Stockwell, First results with an MF radar at McMurdo, Antarctica: characteristics and variability of motions near semidiurnal period, Geophys. Res. Lett., 25, 297-300, 1998.

Hernandez, G., R. W. Smith, and J. Connor, Neutral wind and temperature in the upper atmosphere above South Pole, Antarctica, Geophys. Res. Lett., 19(1), 53-56, 1992 a.

Hernandez, G., R. W. Smith, G. J. Fraser, and W. L. Jones, Largescale waves in the upper mesosphere at Antarctic high latitudes, Geophys. Res. Lett., 19(13), 1347-1350, 1992b.

Hernandez, G., G. J. Fraser, and R. W. Smith, Mesospheric 12-hour oscillations near South Pole, Antarctica, Geophys. Res. Lett., 20(17), 1787-1790, 1993.

Hernandez, G., R. W. Smith, and G. J. Fraser, Antarctic high-latitude mesospheric dynamics, Adv. Space Res., 16(5), 71-80, 1995.

Hernandez, G., J. M. Forbes, R. W. Smith, Y. I. Portnyagin, J. F. Booth, and N. A. Makarov, Simultaneous mesospheric wind measurements near South Pole by optical and meteor radar measurements, Geophys. Res. Lett., 23(10), 1079-1082, 1996.

Hernandez, G., R. W. Smith, J. M. Kelly, G. J. Fraser, and K. C. Clark, Mesospheric standing waves near South Pole, Geophys. Res. Lett., 24, 1987-1990, 1997.

Lindzen, R. S., and D. Blake, Lamb waves in the presence of realistic distributions of temperature and dissipation, J. Geophys. Res., 77, 2166-2176, 1972.

Longuet-Higgins, M. S., The eigen functions of Laplace's tidal equations over a sphere, Philos Trans. R. Soc. London, A262, 511-607, 1968.

Palo, S. E., Y. I. Portnyagin, J. M. Forbes, N. A. Makarov, and E. G. Merzlyakov, Transient long-period waves observed over South Pole, Annales Geophysicae, 16, 1486-1500 1998.

Portnyagin, Y. I., J. M. Forbes, N. A. Makarov, and E. G. Merzlyakov, Main regularities of intradiurnal oscillations in the lower thermosphere over South Pole, Rep. Russ. Acad. Sci., 349(1), 104-105, 1996 (in Russian).

Portnyagin, Y. I., J. M. Forbes, and N. A. Makarov, Unusual characteristics of the lower thermosphere prevailing winds at South Pole, Geophys. Res. Lett., 24(1), 81-84, 1997.

Portnyagin, Y. I., J. M. Forbes, N. A. Makarov, E. G. Merzlyakov, and S. Palo, The summertime 12-hour wind oscillation with zonal wavenumber $s=1$ in the lower thermosphere over the South Pole, Annales Geophysicae, 18, 828-837, 1998.

Sivjee, G. G., and R. L. Walterscheid, Six-hour zonally symmetric tidal oscillations of the winter mesopause over the South Pole Station, Planet. Space Sci., 42, 447-453, 1994.

Stockwell, R. G., L. Mansinha, and R. P. Lowe, Localization of the complex spectrum: the $S$ transform, IEEE Trans. Signal Proc., 44, 998-1001, 1996.

Walterscheid, R. L., G. G. Sivjee, G. Schubert, and R. M. Hamwey, Large amplitude semidiurnal temperature variations in the polar mesosphere: evidence of a pseudotide, Nature, 324, 347-349, 1986.

Whittle P., Hypothesis testing in time series analysis, Uppsala, 1951. 